
\title{
3 Research Square \\ Prime Editor-mediated Correction of a Pathogenic Mutation in Purebred Dogs
}

\section{Dong Ern Kim}

Chungnam National University

Ji Hye Lee

Chungnam National University

Kuk Bin Ji

Chungnam National University

Eun Ji Lee

Chungnam National University

Chuang Li

Chungnam National University

Hyun Ju Oh

Chungnam National University

Kang Sun Park

MK biotech

Seung Hoon Lee

National Institute of Animal Science

Okjae Koo

ToolGen Inc

Min Kyu Kim ( $\nabla$ kminkyu@cnu.ac.kr)

Chungnam National University

\section{Research Article}

Keywords: Domestic dogs, PE, HD, large-sized dogs, surgical intervention

Posted Date: December 29th, 2021

DOI: https://doi.org/10.21203/rs.3.rs-1105540/v1

License: (1) This work is licensed under a Creative Commons Attribution 4.0 International License.

Read Full License 


\section{Abstract}

Canine hip dysplasia (HD) is a multifactorial disease caused by interactions between genetic and environmental factors. HD, which mainly occurs in medium- to large-sized dogs, is a disease that causes severe pain and requires surgical intervention. However, the procedure is not straight-forward, and the only way to ameliorate the situation is to exclude individual dogs with HD from breeding programs. Recently, prime editing (PE), a novel genome editing tool based on the CRISPR-Cas9 system, has been developed and validated in plants and mice. In this study, we successfully corrected a mutation related to HD in Labrador retriever dogs for the first time. We collected cells from a dog diagnosed with HD, corrected the mutation using $\mathrm{PE}$, and generated mutation-corrected dogs by somatic cell nuclear transfer. The results indicate that PE technology can potentially be used as a platform to correct genetic defects in dogs.

\section{Introduction}

Domestic dogs (Canis lupus familiaris) are the most variable mammalian species ${ }^{1,2}$. More than 400 breeds have been developed by intense artificial selection from a limited number of founders ${ }^{1,3}$. Consequently, purebred dogs have a greater risk of suffering from genetic disorders than any other species $^{4}$. A number of scientific publications have described the health problems of purebred dogs $s^{5-11}$ and emphasized the need for action ${ }^{9-14}$; the problem has also been highlighted recently in public media $^{15}$. As a result, many breeders are increasingly using DNA tests to reduce the frequency of deleterious mutations in their breeding programs ${ }^{4}$. However, no direct treatment has been developed to solve these inherent problems. In particular, canine hip dysplasia (HD) is the most common inherited polygenic orthopedic trait in dog, however, there is no ideal medical or surgical treatment yet ${ }^{16}$.

Genome editing tools, such as CRISPR/Cas9 technology, can be a solution to this genetic problem. In particular, prime editing (PE), a novel and universal precision genome-editing technology has great potential for the correction of pathogenic alleles in purebred dogs. Unlike the conventional CRISPR/Cas9 system, PE does not induce double-strand breaks, which can induce random indel mutations at the target locus. PE was designed to generate a nick in single strands of the target genome locus, and then induce accurate target sequence switching using reverse transcriptase ${ }^{17}$. While PE was originally developed in human cells ${ }^{17}$, it has recently been used to develop genome-edited plant varieties and animals, including mice and fruit flies ${ }^{18-20}$. However, there is no report on the use of PE in dogs.

In this study, we used PE to determine whether point mutations causing canine HD can be corrected in canine fibroblasts. Furthermore, we attempted to confirm the possibility of producing genetically modified dogs by somatic cell nuclear transfer (SCNT) using PE-mediated genetically gene-corrected canine fibroblasts.

\section{Results}




\section{Vector construction and prime editor guide RNA design}

We selected the target point mutation locus for PE-mediated gene correction based on our previous study that identified 25 SNPs correlating with hip dysplasia in dogs ${ }^{21}$. Among the 25 SNPs, BICF2S23030416 was selected as the genome editing target in this study because it showed the highest statistical significance for canine HD ( $p<0.0001)$. The BICF2S23030416 SNP is located in an intergenic region on chromosome 4 and is hypothesized to function in regulating MSH homeobox 2 (MSX2). MSX2 has been utilized as a representative marker for cell ossification induction ${ }^{22,23}$. Our previous report showed that dogs (Labrador retrievers) with HD had a T to C point mutation at the BICF2S23030416 locus among the 25 SNP mutations assessed; therefore, we designed a PE vector to correct the $T$ to $C$ point mutation at this target locus. A lentiviral vector expressing both the PE enzyme (CRISPR/Cas9 nickase fused with RNA polymerase) and a prime editor guide RNA (pegRNA) was constructed and cloned (Fig. 1a). The pegRNA consisted of a primer binding site that could hybridize with sequences near the BICF2S23030416 locus, and a reverse transcriptase template containing the corrected genomic sequence at the point mutation site (Fig. 1b).

\section{Establishment of a gene-corrected canine fibroblast cell line}

Ear fibroblasts were collected from a Labrador retriever dog diagnosed with HD (donor patient) and cultured in vitro. The $T$ to $C$ point mutation sequence at the BICF2S23030416 locus in the fibroblasts was determined by sequence analysis (Fig. 1C, donor patient). Lentiviral particles, which is expressing PE, were transduced into the fibroblasts; the rate of transduction was measured by expression of the enhanced green fluorescent protein (EGFP) reporter gene. After 5 days of culture, gDNA was isolated from the transduced fibroblasts. Sequence analysis confirmed that the PE-treated fibroblasts had a T sequence at the BICF2S23030416 locus, mixed with C, indicating that PE successfully recovered one allele of the point mutation at the target site (Fig. 1c).

\section{Generation of gene-corrected dogs}

After showing that we could correct the point mutation in fibroblasts, we demonstrated that we could produce gene-corrected dogs using SCNT. Among the fibroblasts produced using PE, we selected the ' $\mathrm{C}>\mathrm{T}$ cell \#1' for SCNT. In total, 18 reconstructed embryos were generated by SCNT using PE-treated fibroblasts and then surgically transferred into the oviducts of a surrogate mother. Pregnancy was detected by ultrasonography at 40 days of gestation, and two puppies weighing $656 \mathrm{~g}(\mathrm{C}>\mathrm{T}$ dog \#1) and $585 \mathrm{~g}(\mathrm{C}>\mathrm{T}$ dog \#2 were delivered by cesarean section (Fig. 2a). We confirmed the integration of the PE vector by EGFP expression (Fig. 2b) and polymerase chain reaction (PCR) analysis (Fig. 2c). As expected, the $C$ to $T$ gene correction at the BICF2S23030416 locus was confirmed in both puppies (Fig. 2d). These results are in line with the sequence analysis data from the PE-treated fibroblasts used as donor cells for SCNT (Fig. 1c).

\section{Discussion}


In the present study, we successfully generated two gene-corrected dogs, cloned from a dog diagnosed with $\mathrm{HD}$, using PE technology for the first time. $\mathrm{HD}$ is a musculoskeletal disorder caused by an unstable connection between the femoral head and acetabulum and is accompanied by severe pain. It is a common disorder in medium- to large-sized dogs, and is known to cause osteoarthritis, lameness and decreased mobility ${ }^{24}$. HD is a polymorphic disease caused by a combination of genetic and environmental factors ${ }^{25}$. Thus, to reduce the prevalence of HD, breeding strategies incorporating screening schemes are widely used ${ }^{24}$. However, studies that eliminate the cause of HD by directly controlling the causative gene have not yet been reported in dogs.

PE technology is effective and a potential solution for correcting genetic mutations in specific canine breeds. It is a simple and highly efficient gene correction system compared to that of CRISPR/Cas9mediated homology directed repair (CRISPR-HDR) ${ }^{17-19}$. The CRISPR-HDR method is dependent on cell division events and requires an additional donor DNA template to correct genetic mutations. $\mathrm{PE}$ overcomes the shortcomings of CRISPR-HDR; it can be performed at any stage of the cell cycle and does not require additional donor $\mathrm{DNA}^{17}$. Therefore, $\mathrm{PE}$ is expected to be a very useful tool, enabling precise target sequence correction at specific loci in dogs.

We corrected a single SNP from a dog with the HD phenotype. However, due to multiple SNP mutations are contributed to the HD, additional gene correction at the other SNP loci related to HD might be needed to generate a fully HD-recovered canine breed. We regarded the current study is the starting point to overcome HD of purebred dog. Thus, we integrated our PE vector into the genome of our gene corrected dogs and plan to perform further studies focused on correcting the additional SNPs. This will also

provide valuable information for understanding the role of each SNP as it relates to HD. Since canine HD is remarkably similar in clinical expression and pathogenesis to that of human $\mathrm{HD}^{26}$, information gleaned from gene-corrected dogs may be very useful in understanding human HD. Thus, PE may be a very useful tool for generating genome-edited dog models to study human diseases.

In conclusion, we successfully confirmed the feasibility of PE in dogs and produced HD-related genecorrected dogs using PE. To the best of our knowledge, this is the first study to adapt PE for use in a canine system. Further studies to analyze gait, behavior, and mobility of the current gene-corrected dogs, and the generation of additional gene-corrected dogs, are needed to understand the relationship between each SNP and HD.

\section{Materials And Methods}

\section{Ethics statement.}

The experimental procedures and methods used in this study were approved by the Animal Welfare and Ethics Office (2019012A-CNU-174), Chungnam National University, Daejeon, and performed according to "The Guide for the Care and Use of Laboratory Animals" published by IACUC of Chungnam National University. Female mixed dogs from 2 to 6 years of age were used in this study as oocyte donors and 
embryo transfer recipients. The dogs were housed indoors and fed once daily with water ad libitum. All methods and protocols were performed in accordance with relevant guidelines and regulations.

\section{Construction of prime editor vector and production of lentiviral particles.}

The vector for PE was purchased from Addgene (Watertown, MA, USA : \#135955) and modified to correct HD-related SNPs. Briefly, the CMV promoter was obtained by PCR using the primer sets $5{ }^{\prime}-$ gaattcttgacattgattattgactag-3' and 5'-tctagaaatttcgataagccagtaagc-3', and inserted into the vector by $E c o R /$ and $X b a l$ enzyme cuts. The pegRNA targeting the HD locus was newly synthesized and then added to the vector using Pac》 and EcoRI. Finally, the vector was confirmed through sequencing. The lentiviral particles of PE vector were produced by commercial vendor (Lugen SCl, Inc., Bucheon, South Korea).

\section{Collection and establishment of canine fibroblast cell lines, transduction, and transgene analysis.}

Fibroblasts were collected from the ears of an 18-month-old Labrador retriever diagnosed with HD (donor patient). The primary fibroblasts were cultured in vitro using culture medium composed of DMEMGlutaMAX, 15\% fetal bovine serum, and 1\% penicillin/streptomycin solution (GIBCO, Inc.). For transduction, 100 multiplicity of infection (MOI) of the PE lentiviral particles, containing $1 \mu \mathrm{g} / \mathrm{mL}$ of polybrene, was transduced into $1 \times 10^{5}$ fibroblasts per a well of 12-well plate. Transgene expression was confirmed by EGFP and integration of the vector was confirmed by sequence analysis.

\section{SCNT and embryo transfer.}

For generating gene corrected dogs, SCNT followed by embryo transfer was performed following the method described elsewhere ${ }^{27}$. Briefly, in vivo matured oocytes with the first polar body were used for micromanipulation. Metaphase chromosomes were removed by aspiration from the oocytes. A single cell ( $\mathrm{C}>\mathrm{T}$ cell) was transferred into the perivitelline space of an enucleated oocyte, and each donor cellcytoplast couplets were fused by two pulses of direct current (24-26 V for $15 \mu \mathrm{sec})$ using an Electro-Cell fusion apparatus. The fused SCNT embryos were chemical activated by incubating with $10 \mu \mathrm{M}$ calcium ionophore (Sigma) and then $1.9 \mathrm{mM}$ 6-dimethylaminopurine (6-DMAP). The activated SCNT embryos were surgically transferred into the oviducts of oestrus-synchronized surrogates. Pregnancy was confirmed by ultrasonography at 30 days after embryo transfer.

\section{PCR validation and sequencing analysis.}

Transgene integration into the genome of transduced fibroblasts and gene-corrected dogs was confirmed by PCR. The PCR primers used to validate the Cas 9 sequence in the vector were 5 '-catcgctattaccatggtgat$3^{\prime}$ and 5 '-ctcttgcagatagcagatcc-3'. These primer sets detected the linkage between the CMV promoter and dCas 9 of the vector used in this study. Sequencing of the target locus was performed to validate the PEmediated gene correction. The sequencing primers used were 5'-gacgccaagggagcagatatt-3' and $5{ }^{\prime}-$ cctctcttatgagaacagcat-3'. 


\section{Declarations}

\section{Competing interests:}

OJK is employee and shareholder of genome editing company, ToolGen Inc. KSP and MKK are employee and shareholder of genome edited animal company, MK biotech. However, the current study was not financially supported by either company.

\section{Acknowledgements}

This work was carried out with the support of "MK biotech" and "Cooperative Research Program of Center for Companion Animal Research (Project No. PJ01398702)" Rural Development Administration, Republic of Korea.

\section{Author contributions}

DEK and OJK and MKK conceived and designed the study. DEK, JHL, KBJ, EJL, CL and KSP was responsible for performed the experiment. KDE, SHL, OJK and MKK analyzed the data. KDE, OJK and MKK wrote the paper. HJO, OJK and MKK edited and reviewed the manuscript. All authors read and approved the manuscript.

\section{Additional Information}

Competing interests: OJK is employee and shareholder of genome editing company, ToolGen Inc. KSP and MKK are employee and shareholder of genome edited animal company, MK biotech. However, the current study was not financially supported by either company.

\section{References}

1. Ostrander, E. A. et al. Dog10K: an international sequencing effort to advance studies of canine domestication, phenotypes and health. National science review 6, 810-824 (2019).

2. Vilà, C., Maldonado, J. E. \& Wayne, R. K. Phylogenetic relationships, evolution, and genetic diversity of the domestic dog. Journal of Heredity 90, 71-77 (1999).

3. Plassais, J. et al. Whole genome sequencing of canids reveals genomic regions under selection and variants influencing morphology. Nature communications 10, 1-14 (2019).

4. Mellersh, C. DNA testing and domestic dogs. Mammalian Genome 23, 109-123 (2012).

5. Oberbauer, A., Belanger, J., Bellumori, T., Bannasch, D. \& Famula, T. Ten inherited disorders in purebred dogs by functional breed groupings. Canine genetics and epidemiology 2, 1-12 (2015).

6. Nicholas, F. W., Wade, C. M. \& Williamson, P. Disorders in pedigree dogs: assembling the evidence. Veterinary Journal (London, England: 1997) 183, 8-9 (2009).

7. Nicholas, F. W., Crook, A. \& Sargan, D. R. Internet resources cataloguing inherited disorders in dogs. The Veterinary Journal 189, 132-135 (2011). 
8. Ginja, M., Silvestre, A., Gonzalo-Orden, J. \& Ferreira, A. Diagnosis, genetic control and preventive management of canine hip dysplasia: a review. The Veterinary Journal 184, 269-276 (2010).

9. Hedhammar, Å. European strategies to enhance canine genetic health. Eur J Companion Anim Pract 9, 93 (1999).

10. Hedhammar, $\AA$. Actions by FCl and WSAVA to promote canine genetic health. European Journal of Companion Animal Practice 15, 22-25 (2005).

11. McGreevy, P. D. \& Nicholas, F. Some practical solutions to welfare problems in dog breeding. ANIMAL WELFARE-POTTERS BAR-8, 329-342 (1999).

12. Indreb $\emptyset, A$. Breeding healthy dogs-A breeder's perspective. European Journal of Companion Animal Practice 15, 17-21 (2005).

13. Asher, L., Diesel, G., Summers, J. F., McGreevy, P. D. \& Collins, L. M. Inherited defects in pedigree dogs. Part 1: disorders related to breed standards. The Veterinary Journal 182, 402-411 (2009).

14. Summers, J. F., Diesel, G., Asher, L., McGreevy, P. D. \& Collins, L. M. Inherited defects in pedigree dogs. Part 2: Disorders that are not related to breed standards. The Veterinary Journal 183, 39-45 (2010).

15. Hedhammar, Å. A., Malm, S. \& Bonnett, B. International and collaborative strategies to enhance genetic health in purebred dogs. The Veterinary Journal 189, 189-196 (2011).

16. Ginja, M., Gaspar, A. R. \& Ginja, C. Emerging insights into the genetic basis of canine hip dysplasia. Veterinary Medicine: Research and Reports 6, 193 (2015).

17. Anzalone, A. V. et al. Search-and-replace genome editing without double-strand breaks or donor DNA. Nature 576, 149-157 (2019).

18. Liu, Y. et al. Efficient generation of mouse models with the prime editing system. Cell discovery 6, 14 (2020).

19. Jiang, Y.-Y. et al. Prime editing efficiently generates W542L and S621I double mutations in two ALS genes in maize. Genome biology 21, 1-10 (2020).

20. Bosch, J. A., Birchak, G. \& Perrimon, N. Precise genome engineering in Drosophila using prime editing. Proceedings of the National Academy of Sciences 118 (2021).

21. Choi, B. H., Kim, T. H., Lee, S. H. \& Im, S. K. SNP for diagnosing hip dysplasia in dog and uses thereof. Republic of Korea patent 10-2012-0043793 (2013).

22. Ichida, F. et al. Reciprocal roles of MSX2 in regulation of osteoblast and adipocyte differentiation. Journal of Biological Chemistry 279, 34015-34022 (2004).

23. Matsubara, T. et al. BMP2 Regulates Osterix through Msx2 and Runx2 during Osteoblast Differentiation*. Journal of biological chemistry 283, 29119-29125 (2008).

24. Lewis, T. W., Blott, S. C. \& Woolliams, J. A. Genetic evaluation of hip score in UK Labrador Retrievers. PloS one 5, e12797 (2010).

25. Wang, S. et al. Genetic correlations of hip dysplasia scores for Golden retrievers and Labrador retrievers in France, Sweden and the UK. The Veterinary Journal 226, 51-56 (2017). 
26. Zhou, Z. et al. Differential genetic regulation of canine hip dysplasia and osteoarthritis. PloS one 5, e13219 (2010).

27. Lee, J. H. et al. Effect of acteoside as a cell protector to produce a cloned dog. Plos one 11, e0159330 (2016).

\section{Figures}

a

cPPT/CTS

P2A

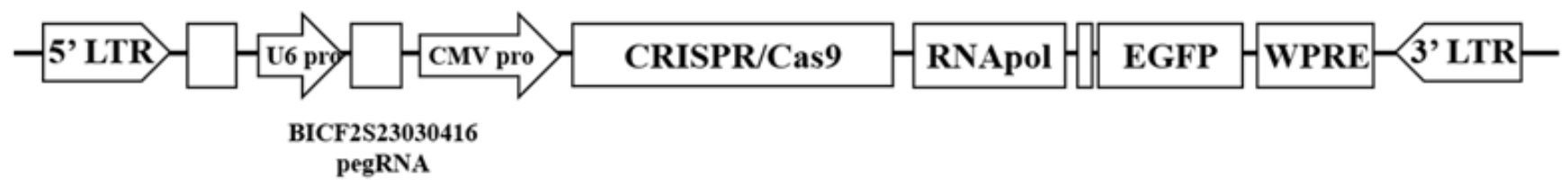

b

\section{GAGGGGAACACACGCAGGAC $[$ scaffold] ACTCTGCTCCTGTCCTGCGTGTGTTCC}

gRNA

RT Template

Primer binding site

c

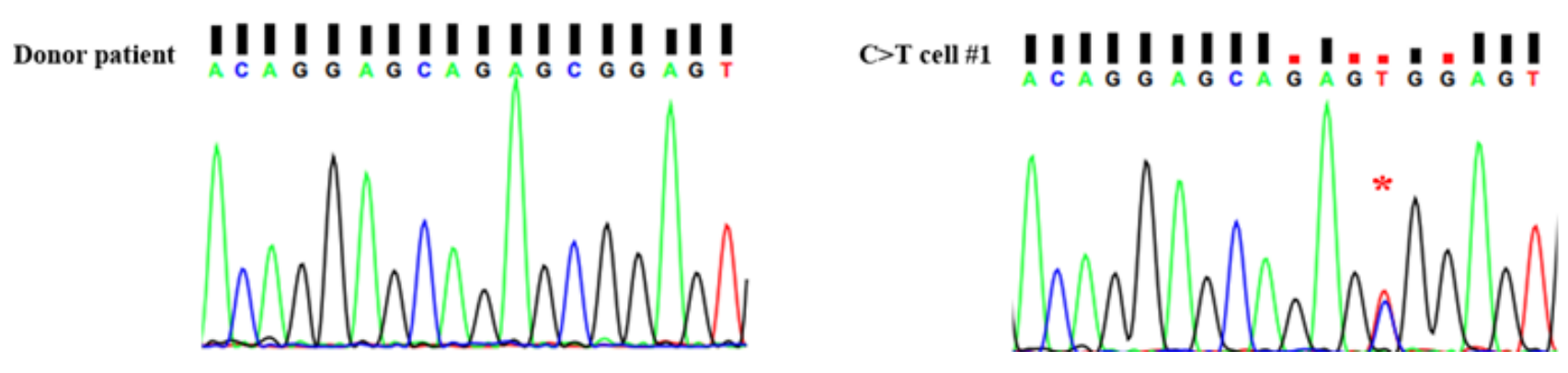

Figure 1

Correction of point mutation in donor cells using prime editor (PE). (a) Schematic of PE vector. It consists of a prime editor that can correct SNP at BICF2S23030416 locus and EGFP as a reporter. (b) Structure and design of PE guide RNA (pegRNA). The bracketed region in orange color indicates the scaffold for pegRNA ${ }^{17}$. The nucleotide $(A)$ in red indicates the SNP mutation site. (c) Chromatographic analysis of the donor patient cells and $\mathrm{C}>\mathrm{T}$ cell $\# 1$. The red asterisk indicates the target locus and confirms the $\mathrm{C}$ to $\mathrm{T}$ sequence correction mediated by $\mathrm{PE}$. 


\section{Figure 2}

Generation and verification of gene-corrected dogs using somatic cell nuclear transfer (SCNT). (a) Bright field and (b) enhanced green fluorescent protein (EGFP) expression under UV light of the gene-corrected dogs. (c) PCR analysis of the vector construct integration in donor patient cells and gene-corrected dogs ( $\mathrm{C}>\mathrm{T}$ dog \#1 and \#2). Only the gene-corrected dogs showed a band at $600 \mathrm{bp}$. (d) Chromatographic analysis of target sequences from the donor patient (cells) and gene-corrected dogs. Blue star asterisks indicate SNP of donor patient and red star asterisks indicate corrected SNP sequence in C>T dogs \#1 and \#2. 